\title{
EPISTEMOLOGICAL OBSTACLES IN COMING TO UNDERSTAND THE LIMIT OF A FUNCTION AT UNDERGRADUATE LEVEL: A CASE FROM THE NATIONAL UNIVERSITY OF LESOTHO
}

Erratum to: Int J of Sci and Math Educ 2009

DOI 10.1007/s10763-008-9143-x

Moru, E. K. (2009). Epistemological obstacles in coming to understand the limit of a function at undergraduate level: A case from the National University of Lesotho. International Journal of Science and Mathematics Education, 7, 431-454.

Due to a technical error, the appendix to the questionnaire design was left out during the production of the article.

In the Methodology section, in the subsection Questionnaire Design the line "Four questions $(2,3,4, \& 5) \ldots$ ". should read: "Four questions $(2,3,4, \& 5$ (see Appendix))...". On the next pages, you will find the appendix.

The online version of the original article can be found at http://dx.doi.org/10.1007/ s10763-008-9143-x. 


\section{APPENDIX}

\section{Question 2}

Two expressions concerning limits are given below.

(a)

$$
\begin{aligned}
& \lim _{x \rightarrow 0} \frac{\sqrt{x^{2}+9}-3}{x^{2}} \quad \text { and } \\
& \text { (b) } \lim _{x \rightarrow \infty} \frac{\sqrt{x^{2}+9}-3}{x^{2}}
\end{aligned}
$$

Answer the questions that follow about expressions (a) and (b).

(i) Is it the same to find the limit of the given function as $x \rightarrow 0$ and as $x \rightarrow \infty$ ? Explain your answer [DOMAIN PROCESS, STEP 3 (a)]

(ii) In finding the limit in question (a) the number 0 is substituted for $x$ in the functional part and the result obtained becomes $\frac{0}{0}$. What conclusion can you draw from this result?

- The limit does not exist.

- The limit is 0 .

- The limit is 1 .

- It is an indeterminate state.

- The limit is $\infty$

- Any other, please specify.

C.hoose the option(s) that best describes your answer.

(iii) For question (b) write down any five numbers which you would substitute for $x$ and explain why you think you have made an appropriate choice of numbers [DOMAIN]

(iv) Calculate the limits of the function as given in (a) and (b). [COORDINATED PROCESS]

\section{Question 3}

You have been asked to find the limits of functions below as $x$ tends to 4 (if any). Which of the statements below would you agree with about the two functions?

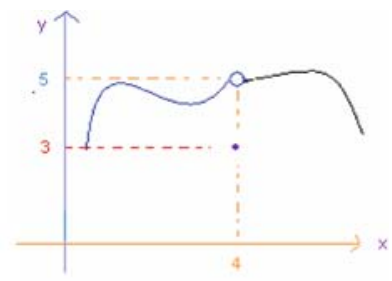

(i)

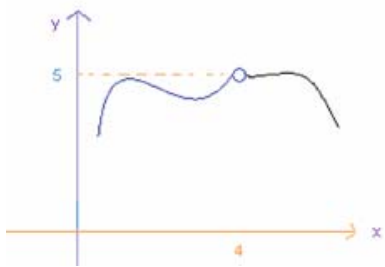

(ii)

(a) In diagram (ii) the limit does not exist since the function is not defined at $x=4$ [ACTION, STEP 1]

(b) In diagram (i) the limit is 3 since it is the function value at $x=4$ [ACTION, STEP 1]

(c) The two functions have the same limit since we are not concerned as to what happens at $x$ $=4$ but to values in its neighbourhood and their function values [COORDINATED PROCESS].

(d) The limits for the two functions cannot be obtained since the two functions are not defined at $x=4$ [ACTION, STEP 1].

Why do you agree with the statement(s) you have chosen? 


\section{Question 4}

How can we see if a function $y=f(x)$ has a limit $L$ as $x$ is approaching 0 ? [COORDINATED PROCESS, STEP 3(c)]]

It is by:

- Calculating $y$ for $x=0$, i.e. calculate $f(0)$ [ACTION, STEP 1];

- Calculating $f(1), f(2), f(3)$ and so on and observe the results [RANGE PROCESS, STEP 3(b)]

- Calculating $f(x)$ for $x=1 / 2,1 / 4,1 / 8$ and so on [RANGE PROCESS, STEP 3(b)];

- Substituting $x$ by 0 in the function formula, and calculate the value [ACTION, STEP 1];

- Substituting numbers that are very close to 0 for $x$ in the formula and look for the value of $y$. [ACTION, STEP 2];

- Substituting numbers that are very close to 0 for $x$ in the formula and look for the value of $y$ that is being approached as $x$ values approach 0 [COORDINATED PROCESS]

(Choose the option(s) that best describes your answer).

Why will you do so?

\section{Question 5}

The function $y=f(x)$ is calculated for values of $x$, and here are some results [ACTION, STEP 2]

\begin{tabular}{|l|l|}
\hline$x$ & $y=f(x)$ \\
\hline 0.7 & 1 \\
\hline 0.74 & 1.8 \\
\hline 0.749 & 1.89 \\
\hline 0.7499 & 1.899 \\
\hline 0.74999 & 1.8999 \\
\hline
\end{tabular}

(a) If this pattern continues what can you say about:

(i) The number $f(x)$ is approaching? [RANGE PROCESS, STEP 3(b)]

(ii) The number $x$ is approaching? [DOMAIN PROCESS, STEP 3 (a)]

(b) Complete the expression below so that it is true about the function represented by the table of values above:

(i) $\quad \lim _{x \rightarrow} f(x)=\ldots$.

[COORDINATED PROCESS, STEP 3(c)]

(ii) After completing the expression above, write in words the meaning of the expression. 